\title{
Cardiac Case Study: Successful Healing Treatment of A 48-Year-Old Male with Block in Heart, Using Yoga Prana Vidya (YPV) Healing System
}

\author{
Ramya Ashwin ${ }^{1}$, Venkata Satyanarayana Nanduri ${ }^{2 *}$ \\ ${ }^{1}$ YPV healer and Researcher, YPV Ashram, Sri Ramana Trust, Thally, Tamilnadu, India \\ ${ }^{2}$ Consultant, Research and Publications, YPV ashram, Sri Ramana Trust, Thally, Tamilnadu, India
}

DOI: $10.36348 /$ sinhc.2019.v02i11.001

*Corresponding author: Venkata Satyanarayana Nanduri
| Received: 27.10.2019 | Accepted: 03.11.2019| Published: 06.11.2019

Email: vsnanduri@yahoo.com

\section{Abstract}

Background: This case study represented a patient's case with a relatively uncomplicated arterial blockage of the Heart, and after prompt Yoga Prana Vidya (YPV) Healing, was successfully treated thereby avoiding costly bypass surgery. Initial medical investigation report stated $90 \%$ block in the arteries of the heart and recommended for open heart surgery. Methods: This is a case study method, going through full detailed records of the patient's health conditions pre and post treatments using YPV healing system observed through lab test records, as well as data collected from follow-up interview of the subject. Results: Analysis of the medical case documents show positive results of improvements obtained with YPV healing method used by healers. Medical investigation after YPV healing confirmed that the arterial blocks disappeared after healing sessions. Conclusion: This case study documents the evidence gathered on the effective uses of YPV in healing and treating arterial blockage. To produce holistic and optimum results, YPV uses a combination of approaches such as, (1) physical exercises including rhythmic yogic breathing, (2) Salt free diet; fruit diet, (3) Meditation techniques, (4) healing by trained and experienced healers, patient participation in group healing, self-healing by patients with regular self-practice of some specified techniques. There is ample scope to conduct further research on the application of YPV as alternatively effective and low cost medicine for various medical conditions.

Keywords: Heart artery blocks, Cardiac bypass surgical Treatment, Yoga Prana Vidya (YPV) healing.

Copyright @ 2019: This is an open-access article distributed under the terms of the Creative Commons Attribution license which permits unrestricted use, distribution, and reproduction in any medium for non-commercial use (NonCommercial, or CC-BY-NC) provided the original author and source are credited.

\section{INTRODUCTION}

Patients with complicated diseases often face a long journey from diagnosis to treatment [1]. The irony of rare diseases is that a lot of people have them. Experience has shown that it is difficult to diagnose, research, and treat rare diseases. Some patients' conditions are also so unique that no proven treatment methods are available to help them recover. Rare diseases are tough to diagnose because most doctors don't see them. "Patients tend to enter into what is called a "diagnostic odyssey," It is really awful for the patients [1]. They go to doctor after doctor. Often this can take years of not knowing what's going on. They start losing hope until they finally get referred to a specialist familiar with the disease." According to Breining [1], about $80 \%$ of rare diseases are genetic, and effective treatment in medical science exists only for a few.

It has been observed that Yoga Prana Vidya ${ }^{\odot}$, (YPV) ${ }^{\circledR}$, which is a non-touch non-drug bio-energy healing method has been used successfully to heal patients having difficult and multiple medical conditions. This paper presents one case study of such cardiac cases successfully healed by $\mathrm{YPV}^{\circledR}$.

\section{Yoga Prana Vidya (YPV)}

According to the founder of Yoga Prana Vidya, the concept of Yoga has to be understood in a broader and in a deeper sense [2]. Yoga in recent times is used to refer to asanas (Postures) and sometimes even to pranayama (a form of breathing). These are parts of yoga and not completely yoga. If viewed from the yogi Patanjali point of view, it is known as Ashtang (eight parts) Yoga. There are many levels of yoga in practice and yoga means all of this. Each of the steps is very important. It is the soul using this physical body but the body is an important vehicle of the soul. This vehicle is so important in the physical world to be maintained, sustained and used for the purpose of the Divine. The purpose of yoga is to achieve union and manifest 
greatness on earth. The incarnated soul or Jeevatma has 3 vehicles.

- The Energy body is called the Pranamaya kosha

- Above that is The Emotional body which is called Kama kosha

- Above that is The Mental body, the Manomaya kosha

To maintain our physical body it involves maintaining the energy body, because energy body is the basic mould of the physical body, which science now recognizes as a bio-Plasmic body. Anything that happens to the physical body, happens to the etheric body. This etheric body is referred to as "etheric double" in theosophy. Any ailment in the physical body has its effect on the etheric body. So we use the term Prana Vidya. Prana is Life Force or Energy used for the maintenance of the energy body and Vidya is basically the technology of how to do so. The technology of maintaining the energy body is Yoga Prana Vidya. Thus YPV gives techniques for maintaining the physical and energy body and also maintain the etheric connectivity with the higher self which involves meditations.

Healing is the process by which the energy body can be renewed thus bringing change in the physical body, because the former interpenetrates the latter. Used up energy or diseased energy can be removed and the energy body can be impregnated with fresh energy. All biological life on earth has the ability to heal itself. Energy follows thought and energy accelerates the healing process. So the energy is used in healing, to accelerate the healing process and the body heals itself.

Healing consists of two processes.

- Cleansing-removal of the used up energy

- Energizing- giving fresh energy.

In YPV, healer becomes a channel of energy who receives and transfers energy. Yoga Prana Vidya (YPV) is a revolutionary form of energy "medicine". It is an ancient science and art that has been revived in a new form which is easily adaptable and in tune with modern day busy life $[3,4]$.

The air around us contains a special life force that keeps us alive. This life force is also known as "prana" ("Breath of Life"). YPV reveals various techniques of using prana in order to promote good health. Yoga Prana Vidya has been used as a tool to deal with psychological and emotional issues also to remedy everyday work and financial stresses of life. It is primarily used as a non-touch, drugless, healing system, complementary (along with) with other healing modalities like Allopathy, Ayurveda, and Homeopathy. However, there are numerous instances where the application of YPV healing alone has been sufficient to alleviate the ailment/ disease (both physical and psychological).

There are many recorded medical cases which have been successfully controlled/cured by YPV healing such as $[5,6]$.

- Diabetes

- Blood pressure and heart ailments

- ENT and respiratory ailments

- Musculoskeletal

- First aid and emergency cases

- Dental cases

- Eye camps

- Cancer cases

- Digestive disorders

- Psychological cases like anxiety, depression, suicidal tendencies, relationship issues, lack of attention and concentration

- Criminal tendencies etc.

For the purpose of this paper, the authors are presenting a heart (arterial) block case which was successfully healed and eliminated the blocks, thereby avoiding a bypass surgery.

\section{CASE REPORT}

Patient background: 48 year old male resident of Hassan, Karnataka state, India.

\section{Pre-YPV Medical History}

Patient did not experience any symptoms of blood pressure or heart ailment prior to this episode. However, he was a known diabetic.

\section{YPV Intervention}

Patient had severe chest pain and burning sensation near chest area. As he was unable to tolerate it he consulted the YPV healer nearby. The Healer called a senior healer immediately after seeing the patient, and the senior healer instructed what healing protocols to be used. Patient thought it was gastric problem and did not suspect it to be some other cause. After initial healing he had some relief but it didn't subside. At this stage the healers suggested to the patient to immediately see a doctor.

He immediately went to a hospital in that town and the emergency doctor took an angiogram. The report (see Figures $1 \& 2$ ) revealed that there was $90 \%$ block in the arteries of heart and recommend open heart surgery. So immediately they shifted patient to a Bangalore hospital as there was no facility for further things in Hassan. After reaching and admitting in Bangalore hospital they did not operate on him immediately because his left hand was swollen (because of being diabetic), and they postponed the surgical operation. 

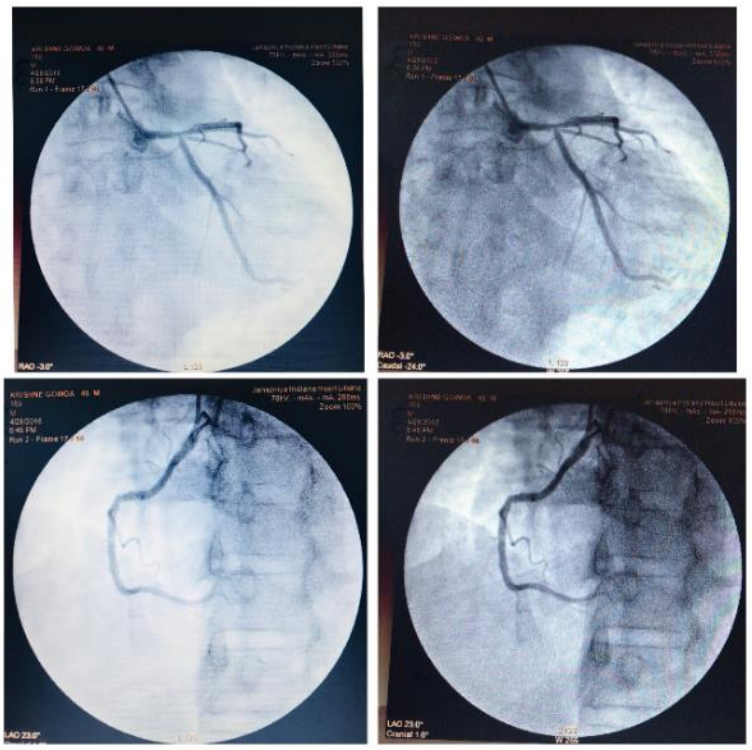

Fig-1: Angiogram before YPV healing

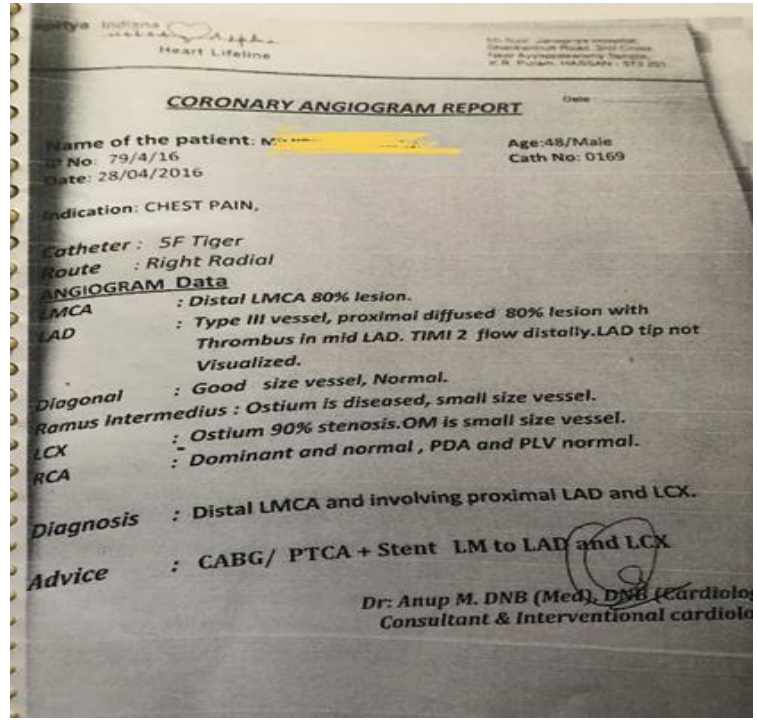

Fig-2: Report Before YPV healing

As the diagnosis revealed that the patient had blocked arteries, and the surgery was postponed, the healers started healing once again as per the request of the patient. The healing team which included a senior most healer supported the required intensive healing.

After five days of observation in the hospital, they took angiogram again before proceeding with surgery. This report stated that (see Figures $3 \& 4$ ) there were no blocks and surgery was not needed. Thus, surgery was avoided and patient became completely normal. Till date his health condition is normal and he is leading a good life.

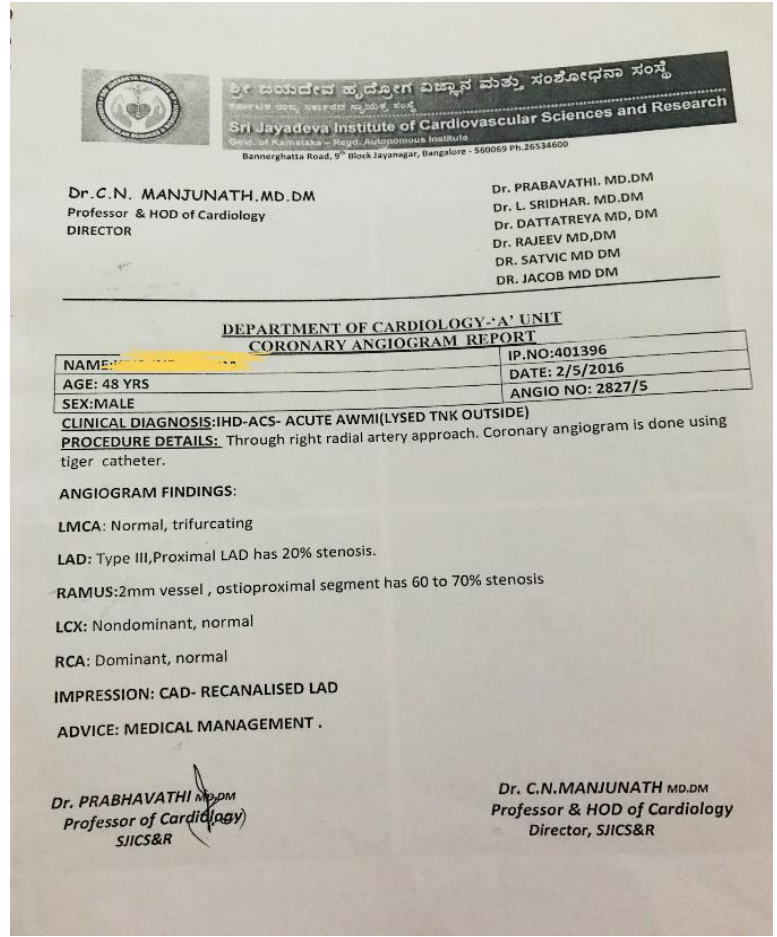

Fig-3: Report after YPV Healing for 5 days

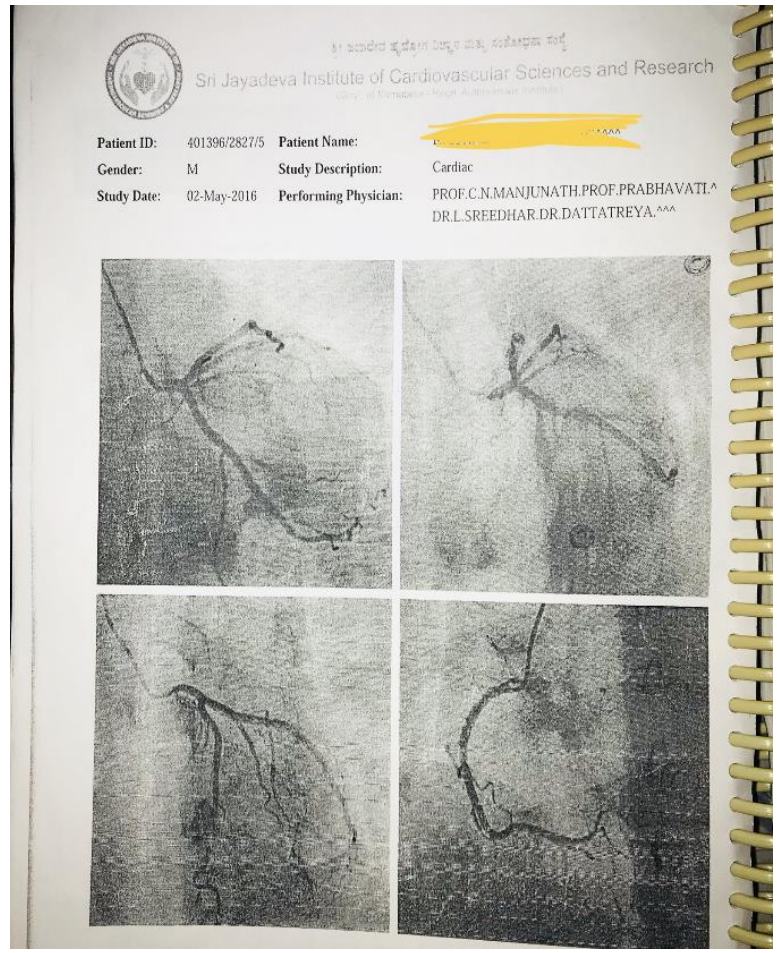

Fig-4: Graphic Status after YPV healing

\section{DISCUSSION}

Coronary bypass surgery redirects blood around a section of a blocked or partially blocked artery in the heart to improve blood flow to the heart muscle. The procedure involves taking a healthy blood vessel from patient's leg, arm or chest and connecting it beyond the blocked arteries in hs/her heart [7]. Although coronary bypass surgery doesn't cure the heart disease that caused the blockages (atherosclerosis or 
coronary artery disease), it can ease symptoms, such as chest pain and shortness of breath. For some people, this procedure can improve heart function and reduce the risk of dying of heart disease.

In contrast to the Coronary bypass surgery, it is observed in this case report that YPV Healing removed blocks in the arteries, which is a non-invasive process. Otherwise, the surgery would have been very costly for the patient, and this YPV Healing enabled saving huge cost to the patient and his family. YPV Healing is a non-invasive and non-touch, no-drug system which is observed to be effective in treatment of this ailment through the evidence shown.

\section{CONCLUSION}

This case report documents the evidence gathered on the effective use of YPV in healing heart ailment. The general conditions under which YPV produces optimum results is also derived from the fact that YPV uses a combination of approaches, (1) physical exercises including rhythmic yogic breathing, (2) Salt free diet; fruit diet, and (3) Meditation techniques, healing by healers, group healing, selfhealing by patients, and regular self-practice by patients. These patients have also become trained healers, practice healing and act as role model for other people with several ailments seeking alternative effective medicine. YPV helps as preventive medicine, when the seekers are receptive and undertake regular practices of the self-practice techniques of YPV such as exercises, Rhythmic breathing, meditation and salt free controlled diet.

\section{ACKNOWLEDGEMENTS}

The authors express sincere thanks to Sri Ramana Trust for giving permission to use their copyright terms, Yoga Prana Vidya ${ }^{\odot}$ and YPV ${ }^{\odot}$, and also for allowing them to use the patient medical reports and data in this report.

\section{REFERENCES}

1. Breining, G. (2017). Rare Diseases Difficult to Diagnose, Cures Hard to Come By. Retrieved from https://news.aamc.org/research/article/rarediseases-difficult-diagnose-cures-hard-come/ 8 January 2019.

2. [2] Yoga Prana Vidya Research. (2019): A compendium of Theory, Research and practice of Yoga Prana Vidya. Unpublished document, YPV Ashram, Sri Ramana Trust, Thally, Tamilnadu, India.

3. Sui, M. C. K. (2015). The ancient science and art of Pranic Healing. Bangalore: Institute of Inner Studies Publishing Foundation India Private Ltd.

4. Sui, M. C. K. (2015). Pranic psychotherapy. Bangalore: Institute of Inner Studies Publishing Foundation India Private Ltd.

5. Rajagopal, A. H., Ashwin, R., \& Nanduri, V. S. (2019). Diabetes Management and Control Using Yoga Prana Vidya (YPV) Healing System. Journal of Biology and Life Science, 10(2), 106-120.

6. Rajagopal, A. H., Ashwin, R., \& Nanduri, V. S. (2019). A study into successful treatment of some difficult Medical cases using Yoga Prana Vidya (YPV) Healing System as alternative medicine. International Journal of Scientific \& Engineering Research, 10(7), 882-887.

7. Mayoclinic.org. (2018). [Online] https://www.mayoclinic.org/testsprocedures/coronary-bypass-surgery/about/pac20384589. 